\title{
Pneumothorax Induced by Computed Tomography Guided Transthoracic Needle Biopsy: A Review for the Clinician
}

This article was published in the following Dove Press journal: International Journal of General Medicine

\author{
Lichuan Zeng (D) \\ Huaqiang Liao' \\ Fengchun Ren' \\ Yudong Zhang' \\ Qu Wang ${ }^{2}$ \\ Mingguo Xie' \\ 'Department of Radiology, Hospital of \\ Chengdu University of Traditional \\ Chinese Medicine, Chengdu, Sichuan \\ Province, People's Republic of China; \\ ${ }^{2}$ Department of Ultrasound, Hospital of \\ Chengdu University of Traditional \\ Chinese Medicine, Chengdu, Sichuan \\ Province, People's Republic of China
}

\begin{abstract}
Percutaneous computed tomography (CT)-guided transthoracic needle biopsy (TTNB) is a valuable procedure for obtaining tissue or cells for diagnosis, which is especially indispensable in thoracic oncology. Pneumothorax and hemoptysis are the most common complications of percutaneous needle biopsy of the lung. According to reports published over the past decades, pneumothorax incidence in patients who underwent TTNB greatly varies. The morbidity of pneumothorax after CT-guided TTNB depends on several factors, including size and depth of lesions, emphysema, the number of pleural surfaces and fissure crossed, etc. Attention to biopsy planning and technique and post-biopsy precautions help to prevent or minimize potential complications. Many measures can be taken to help prevent the progression of a pneumothorax, which in turn might reduce the number of pneumothoraces requiring chest tube placement. A multitude of therapeutic options is available for the treatment of pneumothorax, varying from observation and oxygen treatment, simple manual aspiration, to chest tube placement. When a pneumothorax develops during the biopsy procedure, it can be manually aspirated after the needle is retracted back into the pleural space or by inserting a separate needle into the pleural space. Biopsy side down positioning of the patient after biopsy significantly reduces the incidence of pneumothorax and the requirement of chest tube placement. Aspiration in biopsy side down position is also recommended for treating pneumothorax when simple manual aspiration is unsuccessful or delayed pneumothorax occurred. Chest tube placement is an important treatment strategy for patients with a large or symptomatic pneumothorax. Clinicians are encouraged to understand the development, prevention, and treatment of pneumothorax. Efforts should be made to reduce the incidence of pneumothorax in biopsy planning and post-biopsy precautions. When pneumothorax occurs, appropriate treatment should be adopted to reduce the risk of worsening pneumothorax.

Keywords: pneumothorax, lung biopsy, aspiration, chest tube placement, computed tomography, CT
\end{abstract}

\section{Introduction}

Pneumothorax refers to a medical condition marked by the presence of air in the pleural space, which can be subdivided into spontaneous pneumothoraces and traumatic pneumothoraces according to the aetiology. ${ }^{1}$ Traumatic pneumothoraces, also known as known as iatrogenic pneumothorax, occur as a result of direct or indirect injury to the chest in medical practice. Percutaneous computed tomography-guided TTNB is a well-established and safe investigation technique used to obtain tissue specimens. TTNB includes two basic techniques for sample
Correspondence: Mingguo Xie; Qu Wang Hospital of Chengdu University of

Traditional Chinese Medicine, Chengdu, Sichuan Province, People's Republic of China

Email 767030837@qq.com; 444028177@qq.com
International Journal of General Medicine 2021:14 I013-1022

1013 
acquisition, fine needle aspiration biopsy, and core biopsy. Fine needle aspiration biopsy is applied to extract cells for cytologic evaluation. Core biopsy is adapted with a cutting mechanism that is inserted into an organ or region of interest to extract tissue for histologic evaluation. ${ }^{1}$ It is widely accepted that core biopsy of lung lesions has advantages over the fine needle aspiration biopsy as core biopsy can obtain adequate specimens for histopathology diagnosis. ${ }^{2}$ CT-guided TTNB has been performed in the outpatients as a way of minimizing costs and inconvenience to the patient, providing high diagnostic accuracy, and generating a relatively low complication rate. Pneumothorax is the most frequent and potentially dangerous complication after lung biopsy, despite the majority of patients remaining asymptomatic and not requiring further treatment. ${ }^{3,4}$ The complication of a pneumothorax per se is not highly problematic, but chest pain, shortness of breath, and hypoxia caused by pneumothorax may increase the need for chest tube placement and patient hospitalization.

The prevention and treatment of pneumothorax is a matter of particular interest, and numerous modifications have been evaluated in an attempt to reduce the incidence of pneumothorax and the necessity of chest tube placement. To date, several studies have evaluated the mechanisms of TTNB-related pneumothorax and its management. This article focuses on the current opinion on the prevention and management of pneumothorax after CT-guided biopsy.

\section{Incidence and Etiology}

Pneumothorax is the most common complication after lung biopsy. The rate of pneumothorax varies widely across different studies. The latest meta-analysis ${ }^{4}$ investigated risk factors and pneumothorax rates after percutaneous lung biopsy in 23,104 patients from 36 articles. The pooled overall pneumothorax incidence was $25.9 \%$ (ranging from $4.3 \%$ to $52.4 \%$ ), and the pooled incidence of pneumothorax requiring chest tube placement was $6.9 \%$ (ranging from $0 \%$ to $15 \%$ ). These results are generally in concordance with the guideline of the Society of Interventional Radiology. ${ }^{5}$ The variable incidence is probably caused by various baseline characteristics, biopsy techniques, and analytical methods.

In most cases, a pneumothorax is caused by a traumatic injury to the lung or chest, such as a rib fracture or penetrating injury. Air does not enter into the pleural space because the chest wall and visceral pleura are actual physical barriers to entry. After a lung biopsy, one of the following events is most likely to occur in pneumothorax: ${ }^{6}$ (1) communication between alveolar spaces and pleura, (2) direct or indirect communication between the pleural space and the atmosphere. An unskilled procedure may result in direct or indirect communication between the pleural space and atmosphere, while visceral pleural laceration is regarded as the critical factor in lung biopsy-induced pneumothorax, especially for moderate to a large volume. A pneumothorax can range from asymptomatic to potentially life-threatening based on the size, rate of development, and health of the underlying lung. The majority of cases of small pneumothorax after needle biopsy are asymptomatic and spontaneously resolved. Acute ipsilateral chest pain and dyspnoea are the most common symptoms in the acute presentation of pneumothorax, and with a tension pneumothorax, the patient may become tachycardic and hypotensive and develop cyanosis.

\section{Factors Influencing the Pneumothorax Formation}

Multiple studies have assessed potential risk factors correlated with the development of pneumothorax after TTNBs. The incidence of pneumothorax depends on several factors, where some risk factors are consistent, and others are inconsistent or remain controversial. These factors may be broadly classified into the patient-, and procedure-related factors. The factors that may contribute to the occurrence of pneumothorax are listed in Box 1.

The presence or severity of emphysema, smaller lesion size, and greater lesion depth are associated with a higher likelihood of associated pneumothorax. It is widely accepted that emphysema is the main factor influencing the incidence of pneumothorax after CT-guided needle biopsy, and physicians are reluctant to perform lung biopsy in some cases with outstanding emphysema. However, others have reported that emphysema was not associated with an increased risk for pneumothorax. ${ }^{7}$ It has been suggested that theoretically, smaller lesions with deeper location require more redirection to reach lesions compared with superficial and large lesions, thus causing torque at the pleural surface and expanding the pleural puncture.

According to previous studies, ${ }^{8-10}$ lower lobe location is regarded as a risk factor for pneumothorax compared to the upper and middle lobe location, mainly due to greater 
Box I Factors May Influence the Pneumothorax Formation After CT-Guided Lung Biopsy

\begin{tabular}{|l|}
\hline Patient factors \\
Presence of emphysema \\
Lesion size \\
Lesion depth \\
Lobe location \\
Breath-holding instructions \\
Lesion contact with pleura \\
\hline Procedure factors \\
Experienced operators \\
Number of pleural passes \\
Number of biopsy \\
Traversal of a fissure \\
Needle gauge \\
Needle trajectory angle
\end{tabular}

Notes: In patient-related factors, the presence or severity of emphysema, smaller lesion size, greater lesion depth, and lower lobe location are associated with a higher likelihood of pneumothorax, while breath-holding instructions and lesion contact with pleura are associated with a lower risk. In procedure-related factors, unskilled operators, number of pleural passes/biopsies, traversal of a fissure, greater needle gauge and shallow needle trajectory angle are associated with a higher likelihood of pneumothorax.

respiratory motion of the lower lobe. However, investigators have reported delayed pneumothorax more frequently occurring in upper lobes, ${ }^{11-13}$ which was not consistent with previous studies in which all cases of pneumothorax were included regardless of the onset. In general, lower lobe location with great movement may lead to the early appearance of pneumothorax, and upper lobe location with less movement may result in the late appearance of pneumothorax. Breath-hold after forced expiration is recommended during the biopsy procedure and needle extraction to reduce the movement. ${ }^{14}$

Shiekh et $\mathrm{al}^{7}$ did not report significant differences in the rates of pneumothorax of needle gauge. However, two large patient samples studies by Geraghty et $\mathrm{al}^{15}$ and Kuban et $\mathrm{al}^{16}$ demonstrated a significant reduction in pneumothorax rate when using the smaller coaxial needle with a 19-gauge guide needle versus an 18-gauge needle. Shallow needle trajectory angle was considered as a risk factor for pneumothorax..$^{8,17,18}$ It is believed that the needle at a shallow angle may result in an elongated and larger pleural hole compared to a needle inserted perpendicular to the pleura. Also, the greater angulation might have some connection with fewer attempts in hitting the nodules by a single insertion. The pneumothorax risk increases with an increasing number of pleural puncture passes, the number of biopsies, and traversal of the fissure. $^{7}$ A possible explanation for this finding is that the up-and-down movement of the needle tip during the biopsy procedure results in greater injury to the pleura and more tearing of adjacent lung parenchyma, which may cause an increase in the amount of air leakage. Postbiopsy precautions, such as positioning and refraining from coughing, are also important factors influencing the incidence of pneumothorax. Some researchers have reported that positioning the patient with the biopsy side down after the needle is removed can reduce the rate of overall pneumothorax. ${ }^{19,20}$ It is presumed that "dependent atelectasis" after biopsy side down position prevents air delivery to the pleural-puncture site and also provides a physical barrier to further air leakage after the formation of pneumothorax.

It remains controversial whether age and needle gauge are correlated with the incidence of pneumothorax. On the contrary, gender and type of lesion were not reported to be correlated with the incidence of pneumothorax. Many of the patient or lesion-related factors such as age, lesion depth, and lesion size cannot be changed. Special attention should be paid to the factors influencing the biopsy procedure and post-biopsy precautions.

\section{Preventive Measures Breath-Hold Maneuver}

Multiple maneuvers have been applied according to the risk factors to reduce the incidence of pneumothoraxes during and after lung biopsies. Careful planning is needed to choose the shortest distance from the visceral pleura to the lesion and reduce the number of pleural punctures while crossing a fissure and vessels should be avoided. The deep expiration and breath-hold maneuver is commonly recommended during biopsy procedures. Min et $\mathrm{al}^{21}$ assessed the effect of a breath-hold after forced expiration maneuver on the rate of pneumothorax after TTNB in 440 patients. The breath-hold group had a significantly lower risk of pneumothorax compared with the control group $(8.2 \%$ vs $15.8 \%)$. This technique stabilizes the positions of the target lesions, diaphragm, pleural planes, and fissures, thus ensuring easier access to target lesions. Nevertheless, breath-hold can be extremely difficult during some patients' procedure because of concomitant diseases, stress, or fatigue.

\section{Needle Path Plug}

Injection of various substances like autologous blood, normal saline, or gelfoam into the needle path while 
withdrawing the introducer needle has been reported to successfully reduce post-biopsy pneumothorax. ${ }^{22}$ The incidence of pneumothorax in patients treated with an autologous blood clot seal (ABCS) at the removal of the biopsy sheath widely varies despite the use of similar techniques. This technique was prepared by removing blood from an antecubital vein and injecting it at the level of the biopsy, filling the entire track to the visceral pleura. However, the blood injected into the biopsy track differs from non-clotted blood to clotted blood. Lang et al $^{23}$ reported a different clotted blood technique. Then, $0.5-3.0 \mathrm{~mL}$ of the clot and $0.5-1.5 \mathrm{~mL}$ of supernatant were injected at the level of the biopsied nodule, filling the entire track to the visceral pleura. Pneumothorax occurred in 4 out of the 45 patients $(9 \%)$ who received autologous blood clots and in 14 out of the 32 patients (47\%) without autologous blood clots. The systematic review and meta-analysis conducted by Huo et $\mathrm{al}^{20}$ demonstrated that the blood patch technique by Lang et al had the best reduction in pneumothorax rates, followed by the non-clotted blood and, finally, nonfragmented clotted blood. $\mathrm{Li}$ et $\mathrm{al}^{22}$ performed a prospective, randomized, controlled trial to investigate whether the use of the normal saline tract sealant influence the rate of pneumothorax. The incidence of pneumothorax was $6.2 \%$ in normal saline tract sealant and $26.1 \%$ in the control group. Another prospective study ${ }^{24}$ also demonstrated normal saline tract sealant was an effective technique to reduce the overall pneumothorax incidence and requirement of chest tube placement. Normal physiological saline solution is ubiquitously available, low cost, without adverse reactions, and simple to handle. Those results are consistent with the premise that pulmonary hemorrhage along the needle track represents a protective factor against the development of pneumothorax. ${ }^{3}$ A possible physiological mechanism is that the substances fill the biopsy tract, thus creating a barrier, which prevents air from traveling from the alveoli to the negative pressure zone of the pleural space.

\section{Biopsy/Puncture Site Down Position or Rollover Technique}

In 1982, Zidulka et al ${ }^{25}$ were first to report that placing dogs in the lateral decubitus position with the puncture site down reduced the incidence of pneumothorax after a pleural puncture. In 1990, Cassel et $\mathrm{al}^{26}$ reported the rollover technique, which requires turning the patient in a direction opposite to that of the needle stick immediately after needle removal, significantly reduced the incidence of pneumothorax after percutaneous lung biopsy from $20 \%$ to $5 \%$. Physicians defined this procedure as biopsy/puncture site down positioning or rollover technique. Although some studies have reported that biopsy side down position may not affect the incidence of post-biopsy pneumothorax, ${ }^{27-29}$ much more investigators have reported favorable results. ${ }^{30-32}$ Kinoshita et $\mathrm{al}^{33}$ performed the lung biopsy with a puncture site down position through a window in the CT bed. Although this approach was useful in reducing the rate of pneumothorax, it was not widely utilized because it was ergonomically difficult for the operator as it requires needle manipulation from below the CT bed. Drumm et $\mathrm{al}^{32}$ performed a retrospective study to investigate the relationship between patient position and pneumothorax. In the standard prone or supine group, 184 patients were positioned in prone or supine position depending on the most direct needle path to the lesion, avoiding the fissures. In the biopsy-side down group, 189 patients were positioned in the lateral decubitus position with the biopsy side down for biopsies. Pneumothorax occurred in 50 out of 184 (27.2\%) patients who were positioned in either prone or supine position, and in 20 out of $189(10.6 \%)$ patients who were positioned biopsy side down. These results demonstrate that positioning a patient biopsy side down for lung biopsy can reduce the rate of pneumothorax compared with the standard supine or prone position. However, another study ${ }^{34}$ applying a similar approach showed that an ipsilateral-dependent position did not significantly reduce the pneumothorax rate. In fact, placing the patient in an ipsilateral-dependent position is not always possible compared to the standard prone or supine position. More studies prefer to rapidly rollover patients to the biopsy-side-down position after the needle was removed. It was noted that the time between removal of the needle and placing the patient biopsy-side down position influences the incidence of pneumothoraces. O'Neill et al ${ }^{19}$ published their view on the effect of a rapid needle-out patient-rollover time approach on the rate of pneumothorax after lung biopsy. They conducted a study on 201 patients who underwent percutaneous CT-guided lung biopsy. The first group included 81 biopsies, in which the biopsy needle was immediately removed at the end of the biopsy procedure, and the patient was rolled over onto the stretcher to the biopsy side down position without emphasis on rollover time. In the second group, 120 biopsies were performed with a rapid needle-out patient-rollover time approach as quickly as possible (defined as the time 
between removal of the biopsy needle and placing the patient biopsy-side down). The mean rapid needle-out patient-rollover time in the second group was $9.5 \mathrm{~s}$. A significant decrease in the number of pneumothoraces $(23 \%$ vs $37 \%$ ) and a decrease in the number of drainage catheter insertions was noted in group 2 compared with group 1 (4\% vs $15 \%)$.

Biopsy site-down positioning of the patient after biopsy significantly reduced the incidence of pneumothorax and the requirement of chest tube placement. The pathophysiologic basis for the substantial reduction in the incidence of pneumothorax after puncturesite-down position immediately after needle withdrawal could be complicated. Puncture-site down positioning may be beneficial for the visceral and parietal pleura symphysis and reduction in the size of alveoli, creating a physical barrier to further the leakage of air. Reduction of alveolarto-pleural pressure gradient and accumulation of hemorrhagic fluid around the puncture site was reported to be other two contributing factors. ${ }^{34,35}$ It was suggested that immediately after completing the procedure, patients should be positioned in a "biopsy side down" position for at least 1 hour. It is suggested that the above approaches should be flexibly combined before, during, and after the biopsy procedures to reduce the rate of pneumothorax, thus avoiding chest tube placement.

\section{Management Strategies Conservative Treatment}

Most complications occur immediately or within the first hour after the biopsy. In determining treatment strategies, the size of pneumothorax is less important than the severity of clinical compromise, with breathlessness indicating the need for active intervention. A multitude of therapeutic options is available to reduce the risk of worsening pneumothorax caused by biopsy and the need for chest tube placement. Observation and oxygen supplementation is recommended for patients with limited pneumothorax without symptoms. Observational management has been recommended for stable pneumothorax by both the American College of Chest Physicians (ACCP) and British Thoracic Society (BTS) guidelines. ${ }^{36,37}$ Deep breaths and coughing should be avoided and mobility should be limited after the biopsy. Patients should be supervised if they develop shortness of breath, chest pain, or other symptoms to prevent progression of the pneumothorax. Immediately after the biopsy procedure, a CT scan is performed to determine the presence of the procedure-related complications. Also, patients should undergo follow-up chest radiographs or CT to determine whether the pneumothorax is progressive. It has been suggested that the outpatient can be discharged home when no symptoms of pneumothorax aggravation are noticed after about $6 \mathrm{~h}$ of observation, and the patient should return to the hospital if symptoms developed or diminishing oxygen saturation occurs. Oxygen supplementation at a rate to maintain adequate oxygenation should be used with most pneumothorax patients, as it is beneficial in ameliorating the accompanying hypoxemia that may persist even after drainage of a pneumothorax. It has been reported that oxygen supplementation increases the baseline pleural air reabsorption rate three- to fourfold from $1.25 \%$ per day of the involved hemithorax without supplemental oxygen. ${ }^{38}$ The operators should be able to identify and appropriately manage the complications after lung biopsy procedures.

\section{Simple Manual Aspiration}

In 1996, the simple manual aspiration was first reported by Yankelevitz et $\mathrm{al}^{39}$ as a method for preventing the progression of the large pneumothorax resulting from TTNB, which avoided tube placement in 12 out of 17 patients (70.6\%). Many studies ${ }^{40-42}$ have suggested that simple manual aspiration of post-biopsy pneumothorax performed immediately after biopsy may prevent further progression of pneumothorax and subsequent chest tube placement. BTS guidelines on the management of biopsy-induced pneumothorax suggest initial treatment by aspiration. ${ }^{43}$ The needle from the lesion is retracted back into the pleural space, or a needle is inserted near the initial biopsy site advanced into the pleural space to perform the simple manual aspiration. Manual aspiration, which is effective at preventing progression of the pneumothorax, might exert its effect through the close apposition of the visceral and parietal pleural surfaces after the procedure that create a physical barrier to further leakage of air. When visceral pleural injury is small, the air leak is completely stopped after the aspiration. However, if the closure of a visceral pleural rupture does not occur fast enough for the physical barrier to be created, pneumothorax would develop despite manual aspiration and management with a chest tube would be needed.

Simple manual aspiration has been reported as a safe and effective therapy, whose efficiency greatly varies. ${ }^{35,39}$ There are still many pneumothorax patients who need 
chest tube placement. According to their experience with biopsy side down positioning for prevention of pneumothorax, Yaffe et $\mathrm{al}^{35}$ developed "ipsilateral opposite side aspiration" as a simple, inexpensive new technique for managing pneumothorax. An 18-gauge needle was inserted near the initial biopsy site and advanced into the pleural space for simple aspiration, which was successful in 20 out of the 27 cases. In cases where aspiration was unsuccessful, the patient was reversed to the side opposite the biopsy puncture site, and aspiration was repeated, which was successful in six out of the remaining seven patients. Zeng et al ${ }^{44}$ reported an "opposite position aspiration (from prone to supine or vice versa)" in a larger study sample with an encouraging result. Eighty-two pneumothorax patients were treated with simple manual aspiration that was successful in $58(70.7 \%)$ cases. An opposite position (from prone to supine or vice versa) aspiration was applied to the remaining 24 cases, which was successful in 22 patients, and only two cases needed chest tube placement. Applying the modified method, the total effective rate of aspiration significantly improved from $70.7 \%$ (58/82) to $97.6 \%$ (80/82), thus reducing the use of chest tube placement. Although these two studies were not prospective, randomized controlled studies, the reported results suggested that the biopsy side down aspiration might be an effective treatment for lung biopsy-induced pneumothorax.

The pathophysiologic basis for this is likely due to many factors. ${ }^{44}$ biopsy side down positioning for aspiration may be beneficial for the visceral and parietal pleura symphysis and accumulation of blood within the needle tract, which creates a physical barrier to further leakage of air. This procedure does not increase the alveolar-topleural pressure gradient surrounding the leak region compared to aspiration near the initial biopsy site (Figure 1). Interestingly, Zeng et $\mathrm{al}^{45}$ found that in some pneumothorax patients who have difficulty turning biopsy side down, manual aspiration with puncture site away from initial biopsy one is worth trying to deal with postbiopsy pneumothorax. These results were consistent with the hypothesis of alveolar pressure gradient. A case of successful treatment for pneumothorax in biopsy side down position is shown in Figure 2. The attractiveness of manual aspiration is its potential for an outpatient treatment, minimal invasiveness, and its relative simplicity. This effective procedure should be the first method of treatment as it markedly shortens the length of hospitalization, thus significantly reducing treatment costs.
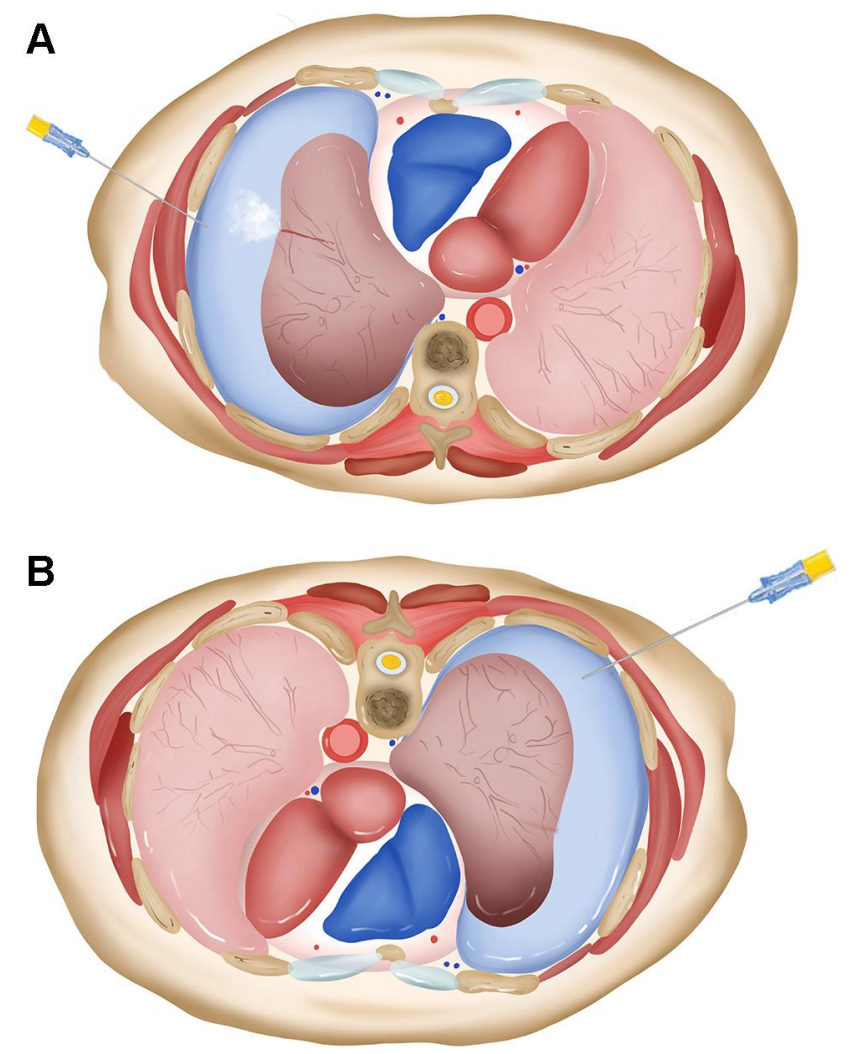

Figure I The model of biopsy side down aspiration for treatment of pneumothorax. (A) Air tends to leak when aspiration is performed near the initial biopsy site because of increased alveolar-to-pleural pressure gradient. (B) The "dependent atelectasis", which occurs in biopsy side down position, provides a physical barrier to further air leakage, and the aspiration does not increase the alveolar-to-pleural pressure gradient around the puncture site.

\section{Management of Delayed Pneumothorax}

While pneumothorax frequently occurs during or immediately after biopsy, TTNBs are suspected and readily diagnosed, serious problems may arise when a pneumothorax presents as a delayed complication. The term "delayed pneumothorax" is not clearly defined. Pneumothorax that develops 3 or 4 hours after TTNB has been defined as delayed pneumothorax in some reports, ${ }^{13,46}$ while other authors suggest that pneumothorax should be considered as "delayed" when it was first detected in follow-up after biopsy. ${ }^{30}$ The risk factor, reason and mechanism of the delayed presentation of pneumothorax are not well known. It is speculated that immediate or delayed occurrence of pneumothorax depends on the severity of injury, the speed of air leakage, and expansibility of the targeted lung and overlying pleura. Some patients had a history of coughing or sudden exertion prior to delayed pneumothorax, which indicated it might be related to the displacement of pleural blood clots formed following the biopsy procedure. Many 

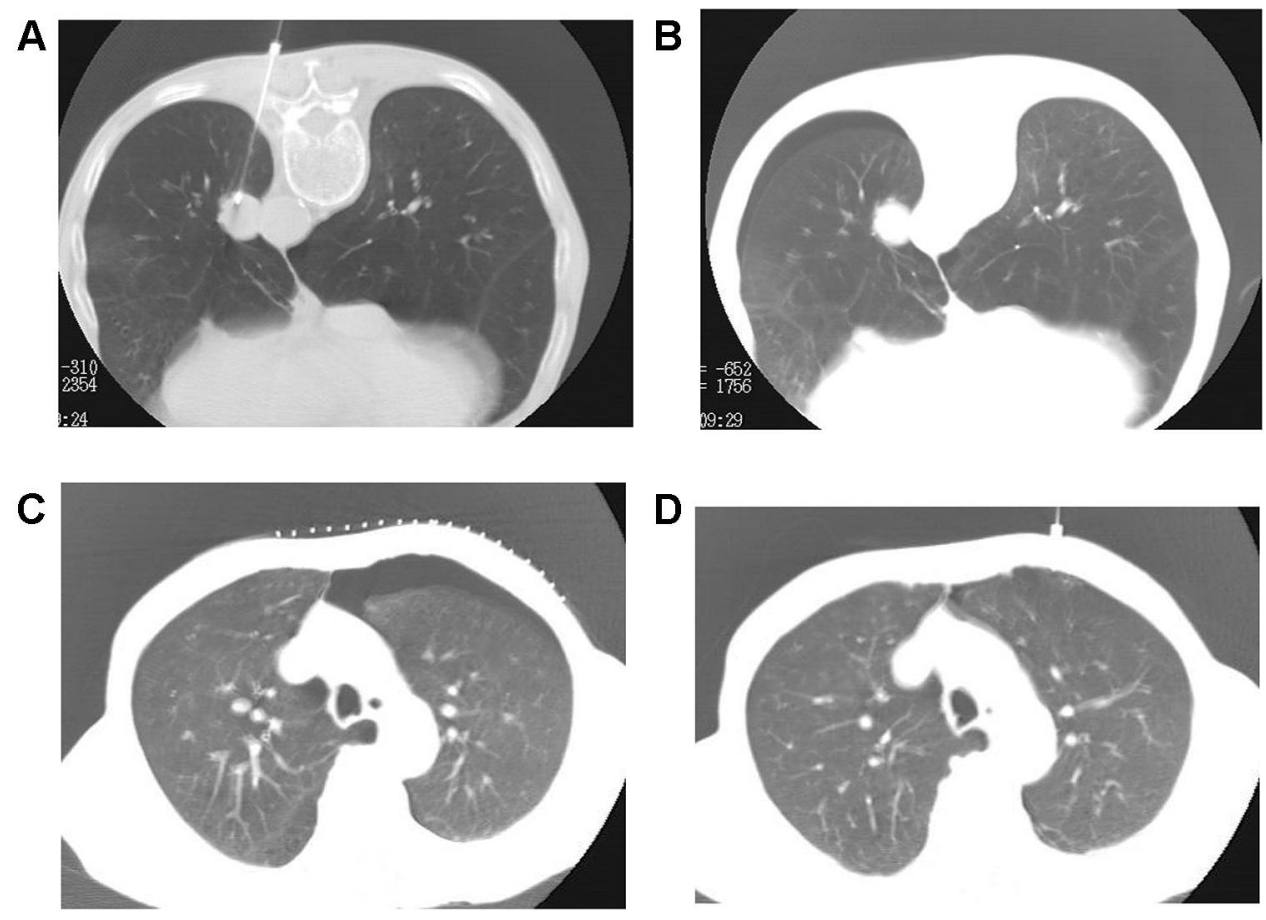

Figure 2 Biopsy side down aspiration approach in a 63-year-old male. (A) A 19-G puncture needle was inserted into the lesion for biopsy. (B) A small amount of pneumothorax was developed. (C) The patient was turned to the biopsy side down position for aspiration when the pneumothorax progressed into moderate or large volume. (D) The pneumothorax almost completely disappeared after the manual aspiration.

operators have found that delayed pneumothorax is not a rare event, with incidence ranging from $2 \%$ to $9 \%{ }^{13}$ Delayed pneumothorax showed higher requirement of chest tube insertion compared to immediate pneumothorax, ranging from $7.1 \%$ to $29.6 \%$ of the overall rate of pneumothorax. ${ }^{11}$ Therefore, the treatment of this condition is clinically significant.

Simple aspiration and chest tube placement are the main maneuvers recommended to deal with the delayed pneumothorax. The influence of puncture site on aspiration as an initial treatment for biopsy-induced delayed pneu-

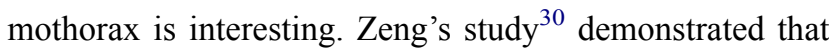
aspiration in the biopsy-side down position had an encouraging result compared to aspiration on the same side as the biopsy. They included 35 patients in group A, for whom aspiration was performed in the same position as the biopsy, and 54 patients in group B, for whom aspiration was performed after biopsy-side down positioning. The overall effective rates were $74.3 \%$ and $92.6 \%$, respectively. This approach is worth trying before chest tube placement in the treatment of delayed pneumothorax. The chest tube placement is recommended when patients suffered from tension pneumothorax, severe dyspnoea and hypoxaemia.

\section{Chest Tube Placement}

The immediate goal of simple aspiration is to eliminate intrapleural air from the pleural cavity and simultaneously reexpand the collapsed lung. Still, this procedure may be insufficient when the parenchymal tear is large and carries a risk of short-term recurrence because it does not promote pleural symphysis. A certain number of patients will require chest tube placement for treatment of pneumothorax. As this management can theoretically irritate the pleura, promoting pleural symphysis, it might have less risk for recurrence than aspiration. ${ }^{47}$ Although chest tube placement has been associated with more complications and a longer stay in hospital than a simple aspiration, it plays an important role in the management of unstable patients with a large or recurrent pneumothorax. When the patient developed tension pneumothorax or suffered from severe dyspnoea and hypoxaemia, chest tube should then be applied for immediate air evacuation followed by recurrence prevention, and patients should be hospitalized. Patients with delayed enlarging pneumothorax are considered to have an air leakage, which has continued beyond the immediate post-biopsy period, and may have higher risk of persistent air leak. Small bore chest tubes are usually sufficient, and large-bore chest tubes are recommended when large air leaks are suspected. 


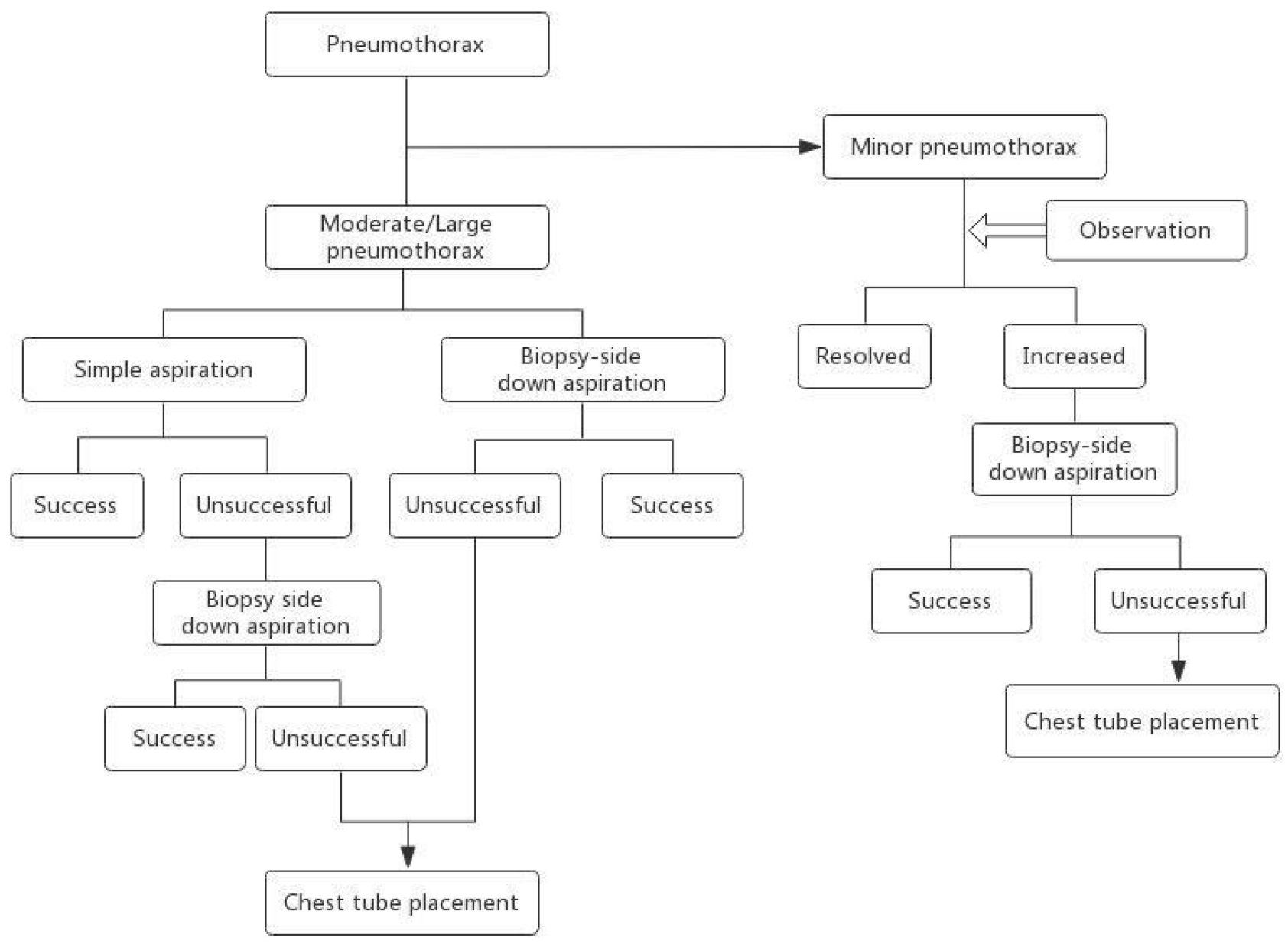

Figure 3 A recommended algorithmic approach to the treatment of lung biopsy-induced pneumothorax.

In general, small and asymptomatic pneumothoraces often do not need any treatment and resolve spontaneously. When the pneumothorax is moderate, or the patient is symptomatic, simple aspiration is required when patient is still on the CT scanner table biopsy side down aspiration is an important supplementary method that is worth trying before chest tube placement. Additional treatment with tube placement should be considered when none of the above methods are effective. The recommended algorithmic approach for the treatment of lung biopsy-induced pneumothorax is shown in Figure 3. The decision is based on a combination of patient symptoms, pneumothorax size, and underlying cardiopulmonary disease.

\section{Summary}

Lung biopsy is a popular and widely accepted procedure for obtaining samples of lung tissue for evaluating an undiagnosed pulmonary nodule or mass. Recent advances in interventional pulmonology have further revolutionized the diagnosis and treatment of lung masses and nodules. Pneumothorax is the most common and sometimes inevitable complication of lung biopsy. It is very important to understand the mechanism, prevention, and treatment of pneumothorax. Accurate planning of the biopsy procedure, which takes into account blood vessels, emphysema, fissures, is associated with a reduction in post-biopsy pneumothoraces. Further simple post-biopsy precautions, such as recumbent positioning, refraining from coughing, and sitting up unassisted are recommended. When pneumothorax occurs, different options are available from observation, aspiration, to chest tube placement. The simple manual aspiration, which enables the removal of air from the pleural cavity, should be used more often. The biopsy side down positioning for aspiration, which offers a solution for patients who remain with resistant pneumothorax after simple aspiration, is worth trying before chest tube placement. Low pneumothorax incidence and 
rate chest placement can be achieved by combining several techniques.

\section{Disclosure}

The authors declare that they have no potential conflicts of interest for this work.

\section{References}

1. Aghajanzadeh M, Asgary MR, Delshad M, et al. Data on the epidemiology, diagnosis, and treatment of patients with pneumothorax. Data Brief. 2018;20:1053-1056. doi:10.1016/j.dib.2018.08.063

2. Anzidei M, Porfiri A, Andrani F, et al. Imaging-guided chest biopsies: techniques and clinical results. Insights Imaging. 2017;8 (4):419-428. doi:10.1007/s13244-017-0561-6

3. Sabatino V, Russo U, D'Amuri F, et al. Pneumothorax and pulmonary hemorrhage after CT-guided lung biopsy: incidence, clinical significance and correlation. Radiol Med. 2020;126(1):170-177. doi:10. 1007/s11547-020-01211-0

4. Huo YR, Chan MV, Habib AR, et al. Pneumothorax rates in CT-guided lung biopsies: a comprehensive systematic review and meta-analysis of risk factors. Br J Radiol. 2020;93(1108):20190866. doi:10.1259/bjr.20190866

5. Gupta S, Wallace MJ, Cardella JF, et al. Quality improvement guidelines for percutaneous needle biopsy. J Vasc Interv Radiol. 2010;21 (7):969-975. doi:10.1016/j.jvir.2010.01.011

6. Noppen M, De Keukeleire T. Pneumothorax. Respiration. 2008;76 (2):121-127. doi: $10.1159 / 000135932$

7. Shiekh Y, Haseeb WA, Feroz I, et al. Evaluation of various patient-, lesion-, and procedure-related factors on the occurrence of pneumothorax as a complication of CT-guided percutaneous transthoracic needle biopsy. Pol $J$ Radiol. 2019;84:e73-e79. doi:10.5114/ pjr.2019.82837

8. Hiraki T, Mimura H, Gobara H, et al. Incidence of and risk factors for pneumothorax and chest tube placement after CT fluoroscopy-guided percutaneous lung biopsy: retrospective analysis of the procedures conducted over a 9-year period. AJR Am J Roentgenol. 2010;194 (3):809-814. doi:10.2214/AJR.09.3224

9. Chami HA, Faraj W, Yehia ZA, et al. Predictors of pneumothorax after CT-guided transthoracic needle lung biopsy: the role of quantitative CT. Clin Radiol. 2015;70(12):1382-1387. doi:10.1016/j. crad.2015.08.003

10. Huang MD, Weng HH, Hsu SL, et al. Accuracy and complications of CT-guided pulmonary core biopsy in small nodules: a single-center experience. Cancer Imaging. 2019;19(1):51. doi:10.1186/s40644019-0240-6

11. Bae K, Ha JY, Jeon KN, Adrish M. Pneumothorax after CT-guided transthoracic lung biopsy: a comparison between immediate and delayed occurrence. PLoS One. 2020;15(8):e238107. doi:10.1371/ journal.pone. 0238107

12. Mills M, Choi J, El-Haddad G, et al. Retrospective analysis of technical success rate and procedure-related complications of 867 percutaneous CT-guided needle biopsies of lung lesions. Clin Radiol. 2017;72(12):1038-1046. doi:10.1016/j.crad.2017.07.023

13. Choi CM, Um SW, Yoo CG, et al. Incidence and risk factors of delayed pneumothorax after transthoracic needle biopsy of the lung. Chest. 2004;126(5):1516-1521. doi:10.1378/chest.126.5.1516

14. Ha JY, Jeon KN, Park MJ, et al. CT-guided biopsy of lung lesions using two needles in difficult and poorly cooperative patients. Springerplus. 2015;4(1):807. doi:10.1186/s40064-015-1614-2

15. Geraghty PR, Kee ST, McFarlane G, et al. CT-guided transthoracic needle aspiration biopsy of pulmonary nodules: needle size and pneumothorax rate. Radiology. 2003;229(2):475-481. doi:10.1148/ radiol.2291020499
16. Kuban JD, Tam AL, Huang SY, et al. The effect of needle gauge on the risk of pneumothorax and chest tube placement after percutaneous computed tomographic (CT)-guided lung biopsy. Cardiovasc Intervent Radiol. 2015;38(6):1595-1602. doi:10.1007/s00270-0151097-0

17. Ko JP, Shepard JO, Drucker EA, et al. Factors influencing pneumothorax rate at lung biopsy: are dwell time and angle of pleural puncture contributing factors? Radiology. 2001;218(2):491-496. doi:10.1148/radiology.218.2.r01fe33491

18. Saji H, Nakamura $\mathrm{H}$, Tsuchida $\mathrm{T}$, et al. The incidence and the risk of pneumothorax and chest tube placement after percutaneous CT-guided lung biopsy: the angle of the needle trajectory is a novel predictor. Chest. 2002;121(5):1521-1526. doi:10.1378/chest.121.5. 1521

19. O’Neill AC, McCarthy C, Ridge CA, et al. Rapid needle-out patient-rollover time after percutaneous CT-guided transthoracic biopsy of lung nodules: effect on pneumothorax rate. Radiology. 2012;262(1):314-319. doi:10.1148/radiol.11103506

20. Huo YR, Chan MV, Habib AR, et al. Post-biopsy manoeuvres to reduce pneumothorax incidence in ct-guided transthoracic lung biopsies: a systematic review and meta-analysis. Cardiovasc Intervent Radiol. 2019;42(8):1062-1072. doi:10.1007/s00270-019-02196-8

21. Min L, Xu X, Song Y, et al. Breath-hold after forced expiration before removal of the biopsy needle decreased the rate of pneumothorax in CT-guided transthoracic lung biopsy. Eur J Radiol. 2013;82(1):187-190. doi:10.1016/j.ejrad.2012.09.013

22. Li Y, Du Y, Luo TY, et al. Usefulness of normal saline for sealing the needle track after CT-guided lung biopsy. Clin Radiol. 2015;70 (11):1192-1197. doi:10.1016/j.crad.2015.06.081

23. Lang EK, Ghavami R, Schreiner VC, et al. Autologous blood clot seal to prevent pneumothorax at CT-guided lung biopsy. Radiology. 2000;216(1):93-96. doi:10.1148/radiology.216.1.r00j13293

24. Billich C, Muche R, Brenner G, et al. CT-guided lung biopsy: incidence of pneumothorax after instillation of $\mathrm{NaCl}$ into the biopsy track. Eur Radiol. 2008;18(6):1146-1152. doi:10.1007/s00330-0080872-6

25. Zidulka A, Braidy TF, Rizzi MC, et al. Position may stop pneumothorax progression in dogs. Am Rev Respir Dis. 1982;126 (1):51-53. doi:10.1164/arrd.1982.126.1.51

26. Cassel DM, Birnberg FA. Preventing pneumothorax after lung biopsy: the roll-over technique. Radiology. 1990;174(1):282. doi:10.1148/radiology.174.1.2294564

27. Collings CL, Westcott JL, Banson NL, et al. Pneumothorax and dependent versus nondependent patient position after needle biopsy of the lung. Radiology. 1999;210(1):59-64. doi:10.1148/radiology.210.1.r99ja1759

28. Tanisaro K. Patient positioning after fine needle lung biopsy-effect on pneumothorax rate. Acta Radiol. 2003;44(1):52-55. doi:10.1258/ rsmacta.44.1.52

29. Kim JI, Park CM, Lee SM, et al. Rapid needle-out patient-rollover approach after cone beam CT-guided lung biopsy: effect on pneumothorax rate in 1191 consecutive patients. Eur Radiol. 2015;25 (7):1845-1853. doi:10.1007/s00330-015-3601-y

30. Zeng LC, Yang HF, Xu XX, et al. Manual aspiration in the biopsy-side down position to deal with delayed pneumothorax after lung biopsy. $J$ Thorac Dis. 2018;10(1):241-246. doi:10.21037/ jtd.2017.12.03

31. Appel E, Dommaraju S, Camacho A, et al. Dependent lesion positioning at CT-guided lung biopsy to reduce risk of pneumothorax. Eur Radiol. 2020;30(11):6369-6375. doi:10.1007/s00330-020-07025-y

32. Drumm O, Joyce EA, de Blacam C, et al. CT-guided lung biopsy: effect of biopsy-side down position on pneumothorax and chest tube placement. Radiology. 2019;292(1):190-196. doi:10.1148/radiol.2019182321

33. Kinoshita F, Kato T, Sugiura K, et al. CT-guided transthoracic needle biopsy using a puncture site-down positioning technique. AJR Am $J$ Roentgenol. 2006;187(4):926-932. doi:10.2214/AJR.05.0226 
34. Leger T, Jerjir N, Gregory J, et al. Does ipsilateral-dependent positioning during percutaneous lung biopsy decrease the risk of pneumothorax? AJR Am J Roentgenol. 2019;212(2):461-466. doi:10. 2214/AJR.18.19871

35. Yaffe D, Shitrit D, Gottfried M, et al. Ipsilateral opposite-side aspiration in resistant pneumothorax after CT image guided lung biopsy: complementary role after simple needle aspiration. Chest. 2013;144 (3):947-951. doi:10.1378/chest.12-2790

36. MacDuff A, Arnold A, Harvey J. Management of spontaneous pneumothorax: British thoracic society pleural disease guideline 2010. Thorax. 2010;65(Suppl 2):i18-i31. doi:10.1136/thx.2010.13 6986

37. Baumann MH, Strange C, Heffner JE, et al. Management of spontaneous pneumothorax: an American college of chest physicians delphi consensus statement. Chest. 2001;119(2):590-602. doi:10.1378/ chest.119.2.590

38. Haynes D, Baumann MH. Management of pneumothorax. Semin Respir Crit Care Med. 2010;31(6):769-780. doi:10.1055/s-00301269837

39. Yankelevitz DF, Davis SD, Henschke CI. Aspiration of a large pneumothorax resulting from transthoracic needle biopsy. Radiology. 1996;200(3):695-697. doi:10.1148/radiology.200.3.8756917

40. Yamagami T, Nakamura T, Iida S, et al. Management of pneumothorax after percutaneous CT-guided lung biopsy. Chest. 2002; 121(4):1159-1164. doi:10.1378/chest.121.4.1159
41. Yamagami T, Kato T, Iida S, et al. Efficacy of manual aspiration immediately after complicated pneumothorax in CT-guided lung biopsy. J Vasc Interv Radiol. 2005;16(4):477-483. doi:10.1097/01. RVI.0000150032.12842.9E

42. Nishiuma T, Ohnishi H, Katsurada N, et al. Evaluation of simple aspiration therapy in the initial treatment for primary spontaneous pneumothorax. Intern Med. 2012;51(11):1329-1333. doi:10.2169/ internalmedicine.51.6919

43. Manhire A, Charig M, Clelland C, et al. Guidelines for radiologically guided lung biopsy. Thorax. 2003;58(11):920-936. doi:10.1136/ thorax.58.11.920

44. Zeng LC, Du Y, Yang HF, et al. Efficacy of an opposite position aspiration on resolution of pneumothorax following CT-guided lung biopsy. Br J Radiol. 2015;88(1051):20150227. doi:10.1259/bjr.20 150227

45. Zeng LC, Liao HQ, Wu WB, et al. Effect of puncture sites on pneumothorax after lung CT-guided biopsy. Medicine (Baltimore). 2020;99(15):e19656. doi:10.1097/MD.0000000000019656

46. Narula N, Siddiqui F, Siddiqui AH, et al. Delayed pneumothorax: a potential complication of transbronchial lung biopsy. Respir Med Case Rep. 2018;23:170-172. doi:10.1016/j.rmcr.2018.02.010

47. Andrivet P, Djedaini K, Teboul JL, et al. Spontaneous pneumothorax. Comparison of thoracic drainage vs immediate or delayed needle aspiration. Chest. 1995;108(2):335-339. doi:10.1378/chest.108.2.335
International Journal of General Medicine

\section{Publish your work in this journal}

The International Journal of General Medicine is an international, peer-reviewed open-access journal that focuses on general and internal medicine, pathogenesis, epidemiology, diagnosis, monitoring and treatment protocols. The journal is characterized by the rapid reporting of reviews, original research and clinical studies

\section{Dovepress}

across all disease areas. The manuscript management system is completely online and includes a very quick and fair peer-review system, which is all easy to use. Visit http://www.dovepress.com/ testimonials.php to read real quotes from published authors. 